\title{
Efficacy of probiotic Bacillus licheniformis DSM 28710 on performance and the mitigation of Clostridium perfringens-induced necrotic enteritis in broiler chickens
}

\author{
W. Van der Veken ${ }^{1 *}$, V. Hautekiet ${ }^{1}$, E.A. Kimminau ${ }^{2}$, C. Hofacre ${ }^{3}$ and G.F. Mathis ${ }^{4}$ \\ ${ }^{1}$ Huvepharma NV, Uitbreidingstraat 80, 2600 Antwerp, Belgium; ${ }^{2}$ Huvepharma Inc., 525 Westpark Drive, Suite 230, \\ Peachtree City, GA 30269, USA; ${ }^{3}$ Southern Poultry Research Group, 1061 Hale Road, Watkinsville, GA 30677, USA; \\ ${ }^{4}$ Southern Poultry Research Inc., 96 Roquemore Road, Athens, GA 30677, USA; woutervanderveken@huvepharma.com
}

Received: 1 July 2020 / Accepted: 29 October 2020

(c) 2020 W. Van der Veken et al.

RESEARCH ARTICLE

BROILERS

\begin{abstract}
The aim of this study was to evaluate the impact of a probiotic Bacillus licheniformis strain (DSM 28710; B-Act ${ }^{\circ}$ ) on growth performance and its capacity to mitigate necrotic enteritis (NE; induced via a Clostridium perfringens challenge) in poultry. A broiler trial was conducted, examining three treatments for 42 days under an induced NE challenge; a negative control (basal diet only); an antibiotic treated group (oxytetracycline hydrochloride (OXT), therapeutic dose of $105 \mathrm{mg}$ OXT/litre in drinking water, for three days after C. perfringens challenge); and a B-Act group (500 g B-Act/tonne of feed, equalling $1.6 \times 10^{12}$ colony forming units B. licheniformis DSM 28710/tonne of feed, supplemented from start until finish). Despite the induced NE challenge, weight gains of the B-Act and OXT groups were similar to each other but significantly higher compared to the control at the end of the study $(P<0.05)$. Weight gain of the B-Act group was already significantly higher compared to the control on day 21 $(P<0.05)$, indicating a potential benefit of the probiotic even before clinical establishment of NE. Feed conversion ratio (FCR) values followed a similar pattern throughout the study, with a significantly lower overall FCR for the B-Act and OXT groups compared to the control $(P<0.05$; d0-42). Birds fed B-Act had significantly $(P<0.05)$ lower NE lesions compared to the control and OXT group on day 21, although OXT was not supplemented to the animals at this stage yet. Both B-Act and OXT groups had significantly $(P<0.05)$ lower NE scores than the control on day 28, demonstrating the effectiveness of the antibiotic treatment and the mitigating effect of B-Act on the effects of a Clostridium perfringens induced NE challenge.
\end{abstract}

Keywords: probiotic, B-Act ${ }^{\oplus}$, necrotic enteritis, poultry, performance, gut health

\section{Introduction}

Both clinical and sub-clinical gastro-intestinal diseases in broilers can lead to major losses, poor welfare and reduced profitability on farm. Necrotic enteritis (NE) is a prominent example, with Clostridium perfringens being a causative agent in the onset and development of the disease. $C$. perfringens is a Gram-positive, spore-forming, anaerobe bacterium and is normally found at levels of less than $10^{5}$ colony forming units (cfu)/g intestinal content in healthy birds (Caly et al., 2015). As such, it can be classified as an opportunistic pathogen, only causing NE if the conditions for bacterial overgrowth are optimal (Williams, 2005).

Seasonal and environmental influences have an impact on NE occurrence, as well as predisposing factors such as but not limited to - nutrition (including protein sources and physical feed forms), the presence of coccidiosis, stress and potential immunosuppression (M'Sadeq et al., 2015). All of these can encourage the growth of C. perfringens, 
resulting in an increase of toxins produced by the bacteria. Consequently, a severe risk to gut health develops, including gut wall integrity (Antonissen et al., 2016). When this happens, the term NE is used to describe the resulting disease, characterised by necrosis and inflammation of the digestive system. NE is often characterised by gut lesions, significantly reduced growth performance, diminished welfare and increased mortality (up to $50 \%$ in clinical cases; Timbermont et al., 2011).

An additional contributing factor to the emergence of NE has been the limitations and bans placed on the use of infeed antibiotics used in sub-therapeutic concentrations (Casewell et al., 2003). This was further highlighted by an epidemiological study in Norway by Kaldhusdal et al. (2016), demonstrating that the removal of such infeed antibiotics in some of the monitored broiler flocks resulted in higher incidences of cholangiohepatitis-related mortality. This post mortem indicator of NE increased from less than $5 \%$ to nearly $10 \%$ in their study, in contrast with the downward trend observed during the previous decade. On an economic level, the total global loss as a consequence of NE outbreaks has previously been estimated to be over $\$ 2$ billion annually (Van der Sluis, 2000), with more recent numbers putting the annual estimation closer to $\$ 6$ billion (Wade and Keyburn, 2015). In an effort to counter these losses, various zootechnical feed additives have been developed to help prevent the development of NE in poultry flocks (M'Sadeq et al., 2015). An interesting example is probiotics, viable micro-organisms which, when administered in adequate amounts, confer health benefits to the host (FAO/WHO, 2002). Their mode of action is often complex, with benefits coming from outcompeting pathogens for nutrients or physical space, producing beneficial compounds, increasing the digestion and/or absorption of nutrients, improving the gut barrier function, reducing gut inflammation and/or interacting with the immune system - or a combination of the above (FAO, 2016).

An example of a well-known genus of probiotics is Bacillus, which has a long history in livestock, and has mainly been used to support the health and functioning of the gastrointestinal tract (Elshaghabee et al., 2017). One species of that genus in particular, Bacillus licheniformis, is of special interest with regard to NE mitigation: the species has been shown to be antagonistic both in vivo and in vitro against C. perfringens (Barbosa et al., 2005; Mingmongkolchai and Panbangred, 2018), restricting one of the major factors in the onset and severity of NE in poultry. This is supported by previous research, in which B. licheniformis probiotics have been recognised as effective probiotics to mitigate enteric challenges (Simon et al., 2001). More recent analysis of the species highlighted this further, confirming its capability to reduce the negative effects caused by NE on the gut microbiota balance of chickens
(Xu et al., 2018). The benefits mentioned above are linked to $B$. licheniformis's mode of action, which is considered to be multifactorial and based on the concept of competitive exclusion. This concept entails not only the direct and indirect competition for nutrients and physical attachment sites in the gut, but also the production of antimicrobial compounds and enhancement of immune system activity (Bernardeau et al., 2017; Caly et al., 2015; Deng et al., 2012; Dischinger et al., 2009).

Based on the available research, the probiotic species has been commercialised in recent years: B-Act ${ }^{\oplus}$ (Huvepharma, Sofia, Bulgaria) is a good example, utilising a single specific B. licheniformis strain (DSM 28710) in its formulation. The probiotic product is marketed globally, including a European registration for use in poultry (EFSA, 2016, 2019), with an effective dose of $1.6 \times 10^{9} \mathrm{cfu}$ per $\mathrm{kg}$ feed. In the following broiler study, the efficacy of B-Act on growth performance and the mitigation of NE, after an induced C. perfringens NE challenge, was investigated. All animals received the same NE challenge and three treatment groups were distinguished: an untreated control (basal diet only), a probiotic group (basal diet $+500 \mathrm{~g} \mathrm{~B}$-Act/tonne of feed) and an antibiotic treated group (basal diet + therapeutic dose of oxytetracycline hydrochloride (OXT) via the drinking water, equal to minimum recommended dose of $22.5 \mathrm{mg}$ OXT/animal/day).

\section{Materials and methods}

\section{Birds and housing}

A total of 1,200, one-day-old healthy male Cobb 500 broilers from the Cobb-Vantress hatchery (Cleveland, GA, USA) were transported to an experimental research facility (Athens, GA, USA). The animals were weighed and randomly allocated to 24 pens, with initial body weights averaging $49 \mathrm{~g}$. There were three treatment groups, each containing eight pens of 50 birds, giving a total of 400 birds per treatment. Each pen had an area surface of 4.65 $\mathrm{m}^{2}$ per pen, containing approximately $10 \mathrm{~cm}$ of built up litter with a top layer of fresh pine shavings. This was to simulate commercial conditions where litter is reused between flocks of broilers, as is common practice in the USA. The trial utilised management systems mimicking commercial conditions as closely as possible. Temperature in the building was optimised via the use of curtain-sided housing (no tunnel ventilation) for the age of the birds. Initially, temperature in the house ranged from $30-32{ }^{\circ} \mathrm{C}$, which was then reduced over time to $23-27^{\circ} \mathrm{C}$ (monitored daily). Fluorescent bulbs above each pen were used for lighting, with 24 hours light from day 1 to day 14, and 18 hours light from day 15 to day 42.

The trial was conducted in accordance with the principles and specific guidelines for animal ethics and welfare, in 
accordance with the Federation of Animal Science Societies policy (FASS). All procedures within the protocol were reviewed and approved by the Institutional Animal Care and Use Committee (IACUC, Southern Poultry Research), including the welfare statement.

\section{Diets and treatments}

Water and feed were available ad libitum throughout the trial period. A commercial basal diet was used, based on maize and soybean meal, and formulated to meet Cobb (2016) commercial requirements. This basal diet was fed in three formulation phases (starter from day 0 to 21; grower from day 21 to 35; finisher from day 35 to 42 ) throughout the trial period. The starter phase was crumbled and initially supplied using a tray, placed on top of the litter in each pen until day 7. From day 8 onwards, the diet was pelleted and fed via a tube-type feeder (one per pen). Table 1 shows the basal diet formulation for each feed phase. Table 2 shows the nutrient analysis for each feed phase.

Three treatments were evaluated, with a first group serving as a negative control and receiving a basal diet only (Control group). The second group was fed the same basal diet, but

Table 1. Composition of diets.

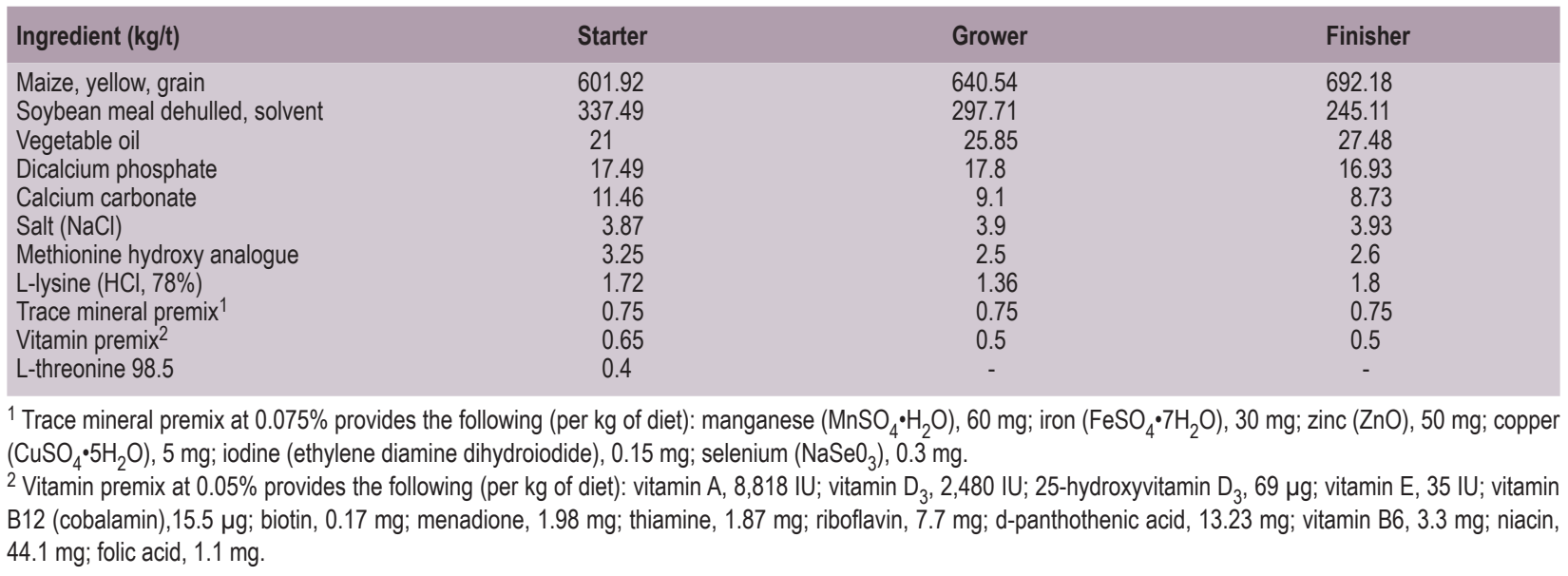

Table 2. Nutrient analysis of the basal diet as formulated in the three different feed phases, represented in percentage (\%, unless otherwise stated; calculated analysis).

\begin{tabular}{|c|c|c|c|}
\hline Nutrient & Starter & Grower & Finisher \\
\hline Dry matter & 88.01 & 87.98 & 87.94 \\
\hline Crude protein & 21.65 & 19.29 & 17.20 \\
\hline Crude fat & 4.48 & 5.10 & 5.39 \\
\hline Crude fibre & 2.18 & 2.11 & 2.05 \\
\hline Calcium & 0.90 & 0.80 & 0.76 \\
\hline Phosphorus (total) & 0.70 & 0.68 & 0.65 \\
\hline Phosphorus (available) & 0.40 & 0.40 & 0.38 \\
\hline Metabolisable energy (kcal/kg) & 3,035 & 3,120 & 3,180 \\
\hline Methionine & 0.61 & 0.54 & 0.52 \\
\hline Lysine & 1.30 & 1.16 & 1.05 \\
\hline Tryptophan & 0.29 & 0.25 & 0.22 \\
\hline Threonine & 0.89 & 0.78 & 0.69 \\
\hline Sodium & 0.18 & 0.18 & 0.18 \\
\hline Potassium & 0.85 & 0.76 & 0.68 \\
\hline Chloride & 0.26 & 0.27 & 0.27 \\
\hline Dig methionine & 0.58 & 0.51 & 0.49 \\
\hline Dig cysteine & 0.30 & 0.27 & 0.25 \\
\hline Dig TSAA & 0.88 & 0.78 & 0.74 \\
\hline Dig lysine & 1.18 & 1.05 & 0.95 \\
\hline Dig tryptophan & 0.29 & 0.25 & 0.22 \\
\hline Dig threonine & 0.78 & 0.68 & 0.60 \\
\hline Dig isoleucine & 1.01 & 0.88 & 0.77 \\
\hline Dig histidine & 0.54 & 0.49 & 0.44 \\
\hline Dig valine & 1.09 & 0.97 & 0.86 \\
\hline Dig leucine & 1.79 & 1.64 & 1.50 \\
\hline Dig arginine & 1.39 & 1.21 & 1.06 \\
\hline Dig phenylalanine & 1.11 & 0.98 & 0.87 \\
\hline
\end{tabular}


supplemented with $500 \mathrm{~g}$ of B-Act per tonne of feed (B. licheniformis DSM 28710, Huvepharma; B-Act group). This equalled $1.6 \times 10^{12} \mathrm{cfu}$ B. licheniformis per tonne feed and was fed continuously throughout all three feed phases, from day 0 to day 42. The third group was also fed the control's basal diet, but received a treatment dose of $105 \mathrm{mg}$ OXT per litre of drinking water (Oxytet ${ }^{\bullet}$ Soluble lot number TSH60008, Huvepharma; OXT group). The antibiotic was supplied via the water system from day 21 to day 24 (after C. perfringens challenge). Based on water intake and body weights, the OXT treatment corresponded with the recommended minimum dose of $22.5 \mathrm{mg}$ OXT/animal/day (according to product label recommendations).

\section{Challenge}

On the day of hatch, all birds were spray vaccinated with a live oocyst vaccine at the recommended label dose (Coccivac-B52 ${ }^{\circ}$ used for coccidiosis management, Merck Animal Health, Madison, NJ, USA) predisposing the animals to induced NE at a later age. Subsequently all birds were administered a C. perfringens challenge on day 19 , 20 and 21. The C. perfringens strain \#6 has been used in previous studies and was isolated from a local commercial broiler operation, known to have caused NE in the past (Hofacre et al., 2003). Each pen received the same amount of inoculum ( $1 \mathrm{ml} / \mathrm{bird}, 50 \mathrm{ml} / \mathrm{pen})$, mixed into the feed at the base of the tube feeder. Fresh inoculum was prepared for each challenge day, with titration levels of $1.0 \times 10^{8-9} \mathrm{cfu} / \mathrm{ml}$.

\section{Measurements}

All birds were individually weighed on the day of hatch and then subsequently weighed by pen on day 21,35 and 42 , to calculate average body weight gain (BWG). Feed intake (FI) was recorded on the same days on a per pen basis, allowing feed conversion ratio (FCR) to be calculated for each growth phase. Pens were checked daily for mortality and no birds were replaced during the study. Birds were only culled to relieve any suffering and all mortalities were recorded. Any culled birds were weighed and FCR was corrected for mortality. Gross necropsy was performed on dead or culled birds, to determine the probable cause of death. Twice daily observations were made for general flock condition, including the availability of feed and water, temperature, any unusual conditions and any abnormal behaviour of the birds.

On day 21 and 28, five birds from each pen were selected at random, euthanised via cervical dislocation, weighed and examined for NE lesions. This was based on the lesion scoring system of Hofacre et al. (1998); score 0: normal; score 1: slight mucus covering small intestine; score 2: necrotic jejunal intestinal mucosa; 3: sloughed and bloody small intestinal mucosa and contents. On day 42 of the study, the remaining birds were weighed by pen and euthanised.

\section{Statistical analysis}

BWG, FI, FCR, NE lesion scores and mortality (total and related to NE) were recorded or calculated, and subsequently analysed. Relevant calculations were corrected for deceased or culled birds. Statistical analysis was performed using STATISTIX 10 for Windows program (Analytical Software, Tallassee, FL, USA). The data was normally distributed and, as such, analysed by ANOVA, with the resulting means separated using Tukey's least significant difference, at a significance level of $P<0.05$.

\section{Results}

Performance measurements of the three groups in terms of FI, FCR and BWG are shown in Table 3.

In the first phase FI was not different between treatment groups ( $\mathrm{d} 0-21 ; P>0.05)$. However, challenging the birds with C. perfringens in the next phase (d19-21) influenced FI heavily, with significantly lower values in the control group compared to the B-Act and OXT groups ( 0 0-35; $P<0.05)$. Considering the full duration of the trial (d0-42), the control group had a significantly lower FI than the B-Act group, with the OXT group's FI not being significantly different to the other two groups $(P<0.05$; Table 3$)$.

Table 3. Feed intake (Fl; kg per pen), feed conversion ratio (FCR) and body weight gain (BWG; in kg) for the three treatments throughout the trial. ${ }^{1}$

\begin{tabular}{|c|c|c|c|c|c|c|c|c|c|}
\hline \multirow[t]{2}{*}{ Treatment $^{2}$} & \multicolumn{3}{|c|}{ Day 0-21 } & \multicolumn{3}{|c|}{ Day 0-35 } & \multicolumn{3}{|c|}{ Day 0-42 } \\
\hline & FI & FCR & BWG & $\mathrm{FI}$ & FCR & BWG & $\mathrm{FI}$ & FCR & BWG \\
\hline $\begin{array}{l}\text { Control } \\
\text { B-Act } \\
\text { OXT }\end{array}$ & $\begin{array}{l}37.4^{\mathrm{a}} \\
36.7^{\mathrm{a}} \\
38.1^{\mathrm{a}}\end{array}$ & $\begin{array}{l}1.461^{a} \\
1.348^{b} \\
1.459^{a}\end{array}$ & $\begin{array}{l}0.47^{\mathrm{a}} \\
0.50^{\mathrm{b}} \\
0.48^{\mathrm{ab}}\end{array}$ & $\begin{array}{l}91.9^{a} \\
101.6^{b} \\
102.0^{b}\end{array}$ & $\begin{array}{l}1.653^{a} \\
1.522^{b} \\
1.487^{b}\end{array}$ & $\begin{array}{l}1.31^{\mathrm{a}} \\
1.55^{\mathrm{b}} \\
1.54^{\mathrm{b}}\end{array}$ & $\begin{array}{l}139.7^{\mathrm{a}} \\
148.5^{\mathrm{b}} \\
145.4^{\mathrm{ab}}\end{array}$ & $\begin{array}{l}1.922^{\mathrm{a}} \\
1.744^{\mathrm{b}} \\
1.666^{\mathrm{c}}\end{array}$ & $\begin{array}{l}1.80^{\mathrm{a}} \\
2.06^{\mathrm{b}} \\
2.03^{\mathrm{b}}\end{array}$ \\
\hline
\end{tabular}

${ }^{1}$ Results with different letter superscripts are significantly different $(P<0.05)$.

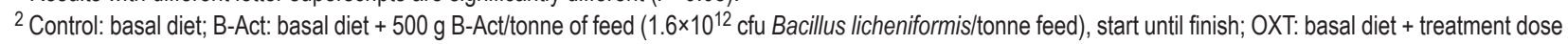
of $105 \mathrm{mg}$ OXT// drinking water, supplied via the drinking water from day 21 to day 24. 
The FCR of the B-Act group was significantly lower than the other two treatment groups in the first phase, indicating an advantage of adding the probiotic to the diet early on $(P<0.05 ; \mathrm{d} 0-21)$. Despite the $C$. perfringens challenge at the end of the first phase, the FCR for the B-Act and OXT groups were similar when evaluated from $\mathrm{d} 0$ to $\mathrm{d} 35$ : both were significantly lower than the control group $(P<0.05$; d0-35). All three diets had significantly different FCR values when evaluated for the complete trial period, with the OXT group achieving the lowest FCR whilst the control group noted the highest FCR $(P<0.05 ; \mathrm{d} 0-42$; Table 3$)$.

The control diet had a significantly lower BWG at the end of the first phase compared to the B-Act group $(\mathrm{d} 21 ; P<0.05)$. After the $C$. perfringens challenge in the following two weeks, the BWG of both the B-Act and OXT groups was significantly higher than the control group, with similar values compared to one another $(P<0.05$; d35; Table 3$)$. The same situation occurred at the end of the trial, with both groups ending the trial with statistically significant higher BWG compared to the control $(P<0.05$; d42; Table 3$)$.

Besides growth performance, Figure 1 illustrates the effect of the control, B-Act and OXT treatments on mortality for the complete trial period. B-Act and OXT groups had significantly lower $(P<0.05)$ mortality than the control, both in terms of NE-associated mortality as well as overall mortality. Mortality due to other reasons than NE included typical challenges for modern broiler production, such as Sudden Death Syndrome (SDS), omphalitis, airsacculitis and so on.

Figure 2 shows NE lesion scores on day 21 and 28. The birds in the B-Act group had significantly $(P<0.05)$ lower lesion scores than the other two treatment groups on day 21 , as the OXT group was not yet treated at this stage. On day

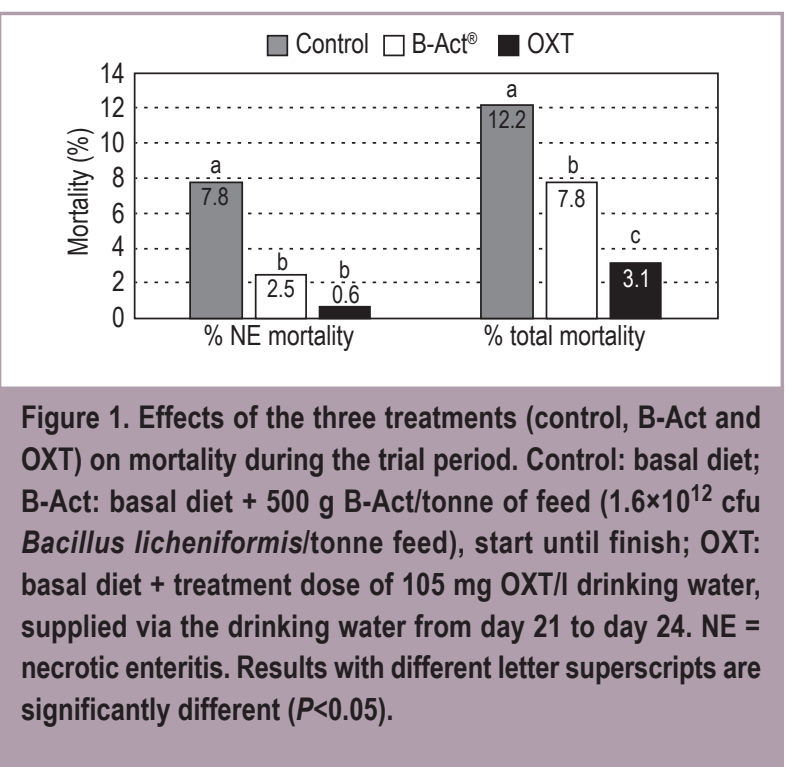

28, OXT and B-Act lesion scores were similar, with both of the scores being lower than in the control group $(P<0.05)$.

\section{Discussion}

The results presented here are in line with previous $B$. licheniformis studies: Knap et al. (2010) conducted three trials, finding that feeding $B$. licheniformis to broilers challenged with $C$. perfringens could mitigate the challenge's negative effects on performance, NE mortality and NE lesion scores. They also included treatments with subtherapeutic levels of virginiamycin (application as growth promotor, $15 \mathrm{~g} /$ tonne feed), recording similar results as for the trialled $B$. licheniformis groups. The research of Zhou et al. (2016) supports these findings further, using a similar challenge model (coccidiosis vaccine followed by C. perfringens inoculation): an increased weight gain and improved FCR for the groups fed probiotic $B$. licheniformis were reported, to the level of being statistically the same as the unchallenged control group. Finally, Lin et al. (2017) also found comparable results in a similarly designed challenge trial, with challenged but $B$. licheniformis supplemented birds achieving statistically similar growth results as the unchallenged control animals. As such, the potential for the probiotic B. licheniformis to support the technical performance of animals under different challenge conditions was demonstrated.

The improvements of NE lesion scores in B. licheniformis supplemented birds adds weight to the topic of gut integrity and epithelial structures, as researched previously. For example, Deng et al. (2012) found that laying hens, kept under heat stress and supplemented with $B$. licheniformis, had improved villi structures and growth performance compared to unsupplemented control animals reared in the same conditions. As proper villi structures ensure optimal

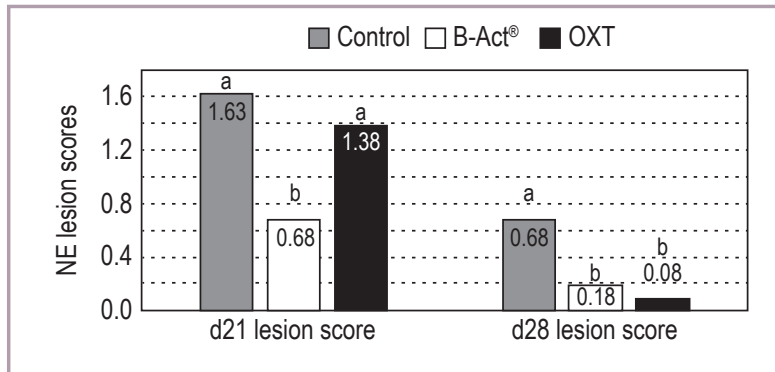

Figure 2. Effects of the three treatments (control, B-Act and OXT) on necrotic enteritis lesion scores on day 21 and 28. Control: basal diet; B-Act: basal diet $+500 \mathrm{~g}$ B-Act/tonne of feed (1.6 $\times 10^{12}$ cfu Bacillus licheniformis/tonne feed), start until finish; OXT: basal diet + treatment dose of $105 \mathrm{mg} \mathrm{OXT/I}$ drinking water, supplied via the drinking water from day 21 to day 24. Results with different letter superscripts are significantly different $(P<0.05)$. 
functioning of the gastrointestinal tract, they play a key role in nutrient absorption and thus growth performance. Other comparable trials showed similar responses, where supplementing diets with $B$. licheniformis supported villi structures, improved growth performance and reduced NE lesion scores (Lei et al., 2013; Lin et al., 2019).

The highest reported mortality levels in the current study were not as large as would have been expected for a major NE challenge, where levels of $50 \%$ have been reported (Timbermont et al., 2011). Nevertheless, probiotic $B$. licheniformis DSM 28710 still significantly reduced mortality compared to the challenge control group, both in terms of NE associated as well as overall mortality.

Apart from being in line with research proving the efficacy of the probiotic species, the current results also confirm earlier work done specifically with $B$. licheniformis DSM 28710 (B-Act), as evaluated by EFSA in 2016 and 2019.

\section{Conclusions}

This trial demonstrates that feeding probiotic B-Act significantly improved growth performance parameters of broilers under a NE challenge. Additionally, NE lesion scores were significantly lower for B-Act supplemented animals, as well as both overall and NE-related mortality. The results achieved with prophylactically administered B-Act were comparable to those realised with therapeutic OXT treatment. As such, continuous administration of B-Act has the potential to be a useful and practical tool to mitigate NE in commercial broilers, contributing to a reduced antibiotic use on-farm as well.

\section{Conflict of interest}

The authors wish to confirm that there are no known conflicts of interest associated with the publication. The research described, including the analysis of the results, was conducted independently and free of any conflicting interests.

\section{References}

Antonissen, G., Eeckhaut, V., Van Driessche, K., Onrust, L., Haesebrouck, F., Ducatelle, R., Moore R.J. and Van Immerseel, F., 2016. Microbial shifts associated with necrotic enteritis. Avian Pathology 45(3): 308-312.

Barbosa, T.M., Serra, C.R., La Ragione, R.M., Woodward, M.J. and Henriques, A.O., 2005. Screening for Bacillus isolates in the broiler gastrointestinal tract. Applied and Environmental Microbiology 71: 968-978.

Bernardeau, M.J., Lehtinen, M.J., Forssten, S.D. and Nurminen, P., 2017. Importance of the gastrointestinal life cycle of Bacillus for probiotic functionality. Journal of Food Science and Technology 54(8): 2570-2584.
Caly, D.L., D'Inca, R., Auclair, E. and Drider, D., 2015. Alternatives to antibiotics to prevent necrotic enteritis in broiler chickens: a microbiologist's perspective. Frontiers in Microbiology 6: 1336.

Casewell, M., Friis, C., Marco, E., McMullin, P. and Phillips, I., 2003. The European ban on growth-promoting antibiotics and emerging consequences for human and animal health. Journal of Antimicrobial Chemotherapy 52: 159-161.

Cobb, 2016. Broiler performance \& nutrition supplement. CobbVantress, Siloam Springs, AR, USA, 14 pp. Available at: https:// tinyurl.com/y4nn8vvg.

Deng, W., Dong, X.F., Tong, J.M. and Zhang, Q., 2012. The probiotic Bacillus licheniformis ameliorates heat stress-induced impairment of egg production, gut morphology and intestinal mucosal immunity in laying hens. Poultry Science 91: 575-582.

Dischinger, J., Josten, M., Szekat, C., Sahl, H.G. and Bierbaum, G., 2009. Production of the novel two-peptide lantibiotic lichenicidin by Bacillus licheniformis DSM 13. PLoS ONE 4(8): e6788. https:// doi.org/10.1371/journal.pone.0006788

Elshaghabee, F.M.F., Rokana, N., Gulhane, R.D., Sharma, C. and Panwar, H., 2017. Bacillus as potential probiotics: status, concerns, and future perspectives. Frontiers in Microbiology 8: 1490. https://doi. org/10.3389/fmicb.2017.01490

European Food Safety Authority (EFSA), 2016. Safety and efficacy of B-Act ${ }^{\oplus}$ (Bacillus licheniformis DSM 28710) for chickens for fattening and chickens reared for laying. EFSA Journal 14(11): 4615.

European Food Safety Authority (EFSA), 2019. Safety and efficacy of B-Act $^{\circledast}$ (Bacillus licheniformis DSM 28710) as a feed additive for turkeys for fattening, turkeys reared for breeding and minor poultry species for fattening or raised for laying. EFSA Journal 17(1): 5536.

Food and Agriculture Organization (FAO), 2016. Probiotics in animal nutrition: production, impact and regulation. Food and Agriculture Organization of the United Nations Technical Report. Food and Agriculture Organization, Rome, Italy, 108 pp. Available at: https:// tinyurl.com/y5k652hh.

Food and Agriculture Organization/World Health Organization (FAO/ WHO), 2002. Guidelines for the evaluation of probiotics in food. Food and Agriculture Organization of the United Nations and World Health Organization Working Group Report. Food and Agriculture Organization, Rome, Italy, 11 pp. Available at: https:// tinyurl.com/y4guddsx.

Hofacre, C.L., Beacorn, T., Collett, S. and Mathis, G., 2003. Using competitive exclusion mannan-oligosaccharide and other intestinal products to control necrotic enteritis. Journal of Applied Poultry Research 12: 60-64.

Hofacre, C.L., Froyman, R., Gautrias, B., Georgie, B., Goodwin, M.A. and Brown, J., 1998. Use of Aviguard and other intestinal bioproducts in experimental Clostridium perfringens-associated necrotizing enteritis in broiler chickens. Avian Diseases 42: 579-584.

Kaldhusdal, M., Benestad, S.L. and Lovland A., 2016. Epidemiologic aspects of necrotic enteritis in broiler chickens - disease occurrence and production performance. Avian Pathology 45(3): 271-274.

Knap, I., Lund, B., Kehlet, A.B., Hofacre, C. and Mathis, G., 2010. Bacillus licheniformis prevents necrotic enteritis in broiler chickens. Avian Disease 54(2): 931-935. 
Lei, K., Li, L., Yu, D.Y., Rajput, L.R. and Li, W.F., 2013. Influence of dietary inclusion of Bacillus licheniformis on laying performance, egg quality, antioxidant enzymes activities and intestinal barrier function of laying hens. Poultry Science 92: 2389-2395.

Lin, E.R., Cheng, Y.H., Hsiao, F.S.H., Proskura, W.S., Dybus, A. and Yu, Y.H., 2019. Optimization of solid-state fermentation conditions of Bacillus licheniformis and its effects on Clostridium perfringensinduced necrotic enteritis in broilers. Revista Brasileira de Zootecnia 48: 0298. https://doi.org/10.1590/rbz4820170298

Lin, Y., Xu, S., Zeng, D., Ni, X., Zhou, M., Zeng, Y., Wang, H., Zhou, Y., Zhu, H., Pan, K. and Li, G., 2017. Disruption in the cecal microbiotia of chickens challenged with Clostridium perfringens and other factors was alleviated by Bacillus licheniformis supplementation. PLoS ONE 12(8): e0182426. https://doi.org/10.1371/journal. pone. 0182426

Mingmongkolchai, S. and Panbangred, W., 2018. Bacillus probiotics: an alternative to antibiotics for livestock production. Journal of Applied Microbiology 124: 1334-1346.

M'Sadeq, S.A., Wu, S., Swick, R.A. and Choct, M., 2015. Towards the control of necrotic enteritis in broiler chickens with in-feed antibiotics phasing-out worldwide. Animal Nutrition 1(1): 1-11.
Simon, O., Jadamus, A. and Vahjen, W., 2001. Probiotic feed effects - effectiveness and expected modes of action. Journal of Animal and Feed Sciences 10(1): 51-67.

Timbermont, L., Haesebrouck, F., Ducatelle, R. and Van Immerseel, F., 2011. Necrotic enteritis in broilers: an updated review on the pathogenesis. Avian Pathology 40(4): 341-347.

Van der Sluis W., 2000. Clostridial enteritis is an often-underestimated problem. World Poultry 16(7): 42-43.

Wade, B. and Keyburn, A., 2015. The true cost of necrotic enteritis. World Poultry 31(7): 16-17.

Williams, R., 2005. Intercurrent coccidiosis and necrotic enteritis of chickens: rational, integrated disease management by maintenance of gut integrity. Avian Pathology 34: 159-180.

Xu, S., Lin, Y., Zeng, D., Zhou, M., Wang, H., Zhou, Y., Zhu,H., Pan, K., Jing, B. and Ni, X., 2018. Bacillus licheniformis normalize the ileum microbiota of chickens infected with necrotic enteritis. Scientific Reports 8: 1744 .

Zhou, M., Zeng, D., Ni, X., Tu, T., Yin, Z., Pan, K. and Jing, B., 2016. Effects of Bacillus licheniformis on the growth performance and expression of lipid metabolism-related genes in broiler chickens challenged with Clostridium perfringens-induced necrotic enteritis. Lipids in Health and Disease 15: 48. https://doi.org/10.1186/s12944016-0219-2 
